\title{
Major Cys protease activities are not essential for senescence in individually darkened Arabidopsis leaves
}

\author{
Adriana Pružinská ${ }^{1,3}$, Takayuki Shindo ${ }^{1}$, Sherry Niessen², Farnusch Kaschani ${ }^{1}$, Réka Tóth ${ }^{5}$, A. Harvey Millar ${ }^{3}$ \\ and Renier A. L. van der Hoorn ${ }^{1,4^{*}}$
}

\begin{abstract}
Background: Papain-like Cys Proteases (PLCPs) and Vacuolar Processing Enzymes (VPEs) are amongst the most highly expressed proteases during leaf senescence in Arabidopsis. Using activity-based protein profiling (ABPP), a method that enables detection of active enzymes within a complex sample using chemical probes, the activities of PLCPs and VPEs were investigated in individually darkened leaves of Arabidopsis, and their role in senescence was tested in null mutants.

Results: ABPP and mass spectrometry revealed an increased activity of several PLCPS, particularly RD21A and AALP. By contrast, despite increased VPE transcript levels, active VPE decreased in individually darkened leaves. Eight protease knock-out lines and two protease over expressing lines were subjected to senescence phenotype analysis to determine the importance of individual protease activities to senescence. Unexpectedly, despite the absence of dominating PLCP activities in these plants, the rubisco and chlorophyll decline in individually darkened leaves and the onset of whole plant senescence were unaltered. However, a significant delay in progression of whole plant senescence was observed in aalp-1 and rd21A-1/aalp-1 mutants, visible in the reduced number of senescent leaves.
\end{abstract}

Conclusions: Major Cys protease activities are not essential for dark-induced and developmental senescence and only a knock out line lacking AALP shows a slight but significant delay in plant senescence.

Keywords: Senescence, Activity-based protein profiling, Papain-like proteases, Vacuolar processing enzymes

\section{Background}

Senescence is the final stage in the development of cells, tissues, and organs and in the case of monocarpic species, entire plants. Leaf senescence is characterized by extensive protein degradation that enables remobilisation of nutrients, especially nitrogen, for use in other parts of the plant, such as newly developing organs, seeds or storage tissues. Protein degradation during senescence involves the disassembly and degradation of the photosystem and metabolic pathways and all other proteins of the living cell until no proteins remain for recycling [1]. Four pathways of chloroplast breakdown

\footnotetext{
* Correspondence: renier.vanderhoorn@plants.ox.ac.uk

'The Plant Chemetics laboratory, Max Planck Institute for Plant Breeding Research, 50829 Cologne, Germany

${ }^{4}$ The Plant Chemetics Laboratory, Department of Plant Sciences, University of Oxford, OX1 3RB Oxford, UK

Full list of author information is available at the end of the article
}

have been identified in Arabidopsis. These pathways involve autophagy, senescence-associated vacuoles (SAVs), chloroplast vesiculation, and selective chloroplast destruction via a 13-lipoxygenase [2-5]. However, despite the importance of this process, the proteases responsible have not all been identified and characterized.

Gene expression studies indicated that Cys proteases are amongst the most abundant proteases during leaf senescence [6]. Senescence-associated Cys proteases are papain-like Cys proteases (PLCPs, protease family C1A in MEROPS Database [7]), legumains or vacuolar processing enzymes (VPEs, family C12), metacaspases (family C14), calpains (family C2) and proteases related to ubiquitin-dependent pathways (families C13, C19 and C85) $[6,8-15]$. Reduced protein degradation in senescing leaf segments of wheat can be achieved upon treatment with Cys protease inhibitors, indicating the involvement of Cys proteases in senescence [16]. 
PLCPs are the major enzymes associated with bulk protein degradation during senescence [14]. However, PLCPs are involved also in other physiological processes such as germination and plant defence [17]. Arabidopsis AALP (SAG2) and SAG12, both encoding PLCPs, are used as standard markers of leaf senescence [18-20]. SAG12 is exclusively expressed in senescent leaves and the encoded protein is localized to senescence-associated vacuoles (SAVs) [2]. By contrast, AALP (SAG2) transcription occurs in leaves at all developmental stages but increases during natural and stress related senescence in (reviewed in [8]). In sweet potato, increased expression of two PLCPs, SPCP2 and SPCP3 (homologs of RD19 and RD21A, respectively) occurs in both natural and stress-induced senescing tissues $[21,22]$ similarly to a soybean PLCP called GMCP3 [23]. Additionally, four putative cDNAs encoding PLCPs (BoCP1, BoCP2, BoCP3, BoCP4) have been isolated from senescing broccoli floret tissue that are similar to Arabidopsis RD19 and RD21A [24]. Arabidopsis RD21A was found in the vacuoles of senescing leaves and is synthesized as a $57-\mathrm{kDa}$ precursor that is slowly processed into a $33-\mathrm{kDa}$ mature protein $(\mathrm{mRD} 21 \mathrm{~A})$ via a $38-\mathrm{kDa}$ intermediate (iRD21A) [25]. These intermediates accumulate in the vacuole as aggregates, however during leaf senescence they are released as a soluble protease upon removal of the granulin domain [25]. In a similar manner, SoCP is a $41-\mathrm{kDa}$ protease with a granulin domain that is transcriptionally induced in senescent leaves of Spinacia oleraceae [26].

In Arabidopsis, VPEs mediate processing of vacuolelocalised proteins during seed germination and developmental or pathogen-mediated programmed cell death [27-30]. It has been proposed that $\gamma \mathrm{VPE}$ might also activate proteases involved in protein recycling during senescence [27]. Transcript levels of $\gamma V P E$ was increased in leaves during development of Arabidopsis [31]. Moreover, $\gamma V P E$ is highly induced in petals of tobacco as they progress in development and it was suggested using it as a senescence marker for petal senescence [32].

Protease activity is regulated by transcriptional and translational processes, but also by post-translational modifications and by protease inhibitors [33]. PLCPs are synthesized with an autoinhibitory prodomain that must be proteolytically removed to activate the enzymes [34]. Senescence-related PLCPs with granulin domain in complex with cystatin have been purified from leaves of spinach and this protease was activated by releasing cystatin from the complex [35]. Similarly, the role of cystatins in modulating of cysteine protease activity during senescence is proposed in barley [36]. Overexpression of rice cystatin in tobacco inhibits Cys protease activity, delaying the decline of Rubisco and two Rubisco activase proteins [37]. AtSerpin1 interacts with RD21A and it is expected that other serpins might regulate senescence [38].

Because of this post-translational regulation, accumulation of proteases or protease-encoding transcripts does not necessarily correlate with protease activity. To study protease activities, rather than transcript or protein accumulation, we applied activity-based protease profiling (ABPP). ABPP is based on the use of fluorescent or biotinylated chemical probes that react irreversibly with the active site of enzymes in a mechanism-dependent manner [39-41]. Here, we applied ABPP to study protease activities during leaf senescence induced by individually darkening leaves of Arabidopsis and we used PLCP and VPE mutants and over expressing lines to confirm the origin of these signals and determine the relative contribution of these proteases to leaf senescence.

\section{Methods}

\section{Plant material and growth conditions}

All Arabidopsis thaliana transgenic and knockout lines were Columbia ecotype and are summarized in Additional file 1: Table S2. The $r d 21-1$, aalp-1, sag12-1, and ctb3-1 mutants [42]; the $r d 21-1 /$ aalp-1 double mutant [43]; the $\gamma \mathrm{VPE}$ overexpressor (35S:: $\gamma V P E$, [44]), the VPE quadruple knockout (qvpe) mutant lacking all four VPEs [45], and the triple mutant $c t b 1 / 2 / 3$ (line \#65-4, [46]) have been described previously. The 35S::RD21 overexpressor line was generated by transforming Col-0 with pRH628 [43] using the flowerdip method. Transgenic plants were selected on kanamycin and homozygous lines were characterized by ABPP (Additional file 2: Figure S3). Plants were grown for six or eight weeks in controlled growth cabinets. Three sets of growth conditions were used: $12 /$ 12 hours day/night cycle at $24{ }^{\circ} \mathrm{C} / 20{ }^{\circ} \mathrm{C}$ temperatures, $16 /$ 8 hours day/night cycle at $22{ }^{\circ} \mathrm{C} / 18{ }^{\circ} \mathrm{C}$ hours (long day), and $8 / 16$ day/night cycle at $22^{\circ} \mathrm{C} / 18^{\circ} \mathrm{C}$ (short day).

\section{Chlorophyll quantification}

A Soil Plant Analysis Development (SPAD) meter (502 Plus Chlorophyll Meter, Spectrum Technologies) was used to determine the relative chlorophyll content [47]. The SPAD analyser measures leaf transmission at two wavelengths (650 and $940 \mathrm{~nm}$ ). Measurements were always taken from the top of the leaf and the values for the five largest rosette leaves were averaged. Eight replicate plants were analysed per treatment. Wild type and the mutant plants were grown in the same tray under same growth conditions. Student's paired t-test, with a two-tailed distribution was used to analyse data.

\section{Senescence assays and other morphological traits during development}

The onset of whole plant senescence was defined as the day on which the number of green leaves started to 
decline [48, 49]. Leaves were classified as senescent when more than half of the leaf area was yellow; otherwise leaves were classified as green. 16 replicate plants were analysed. Both mutant and wild-type were randomly distributed in the same tray. Student's paired t-test, with a two-tailed distribution was used to analyse data.

\section{Semiquantitative RT-PCR}

RNA was extracted from leaves using the Qiagen RNeasy kit. After DNA digestion with TURBO DNase (Ambion), first-strand cDNA was synthesized using SuperScript III reverse transcriptase (Invitrogen). PCR was performed for 30 - 35 cycles with gene-specific primers as follows for SGR1(At4g22920): SGR1-L, 5'-a caagttcccatctccatgc-3'; and SGR1-R, 5' -ggaaaatgtcgcttc acgtt-3'. For SAG12 (At5g45890): SAG12-L, 5'-tccttac aaaggcgaagacg-3'; and SAG12-R, 5' -tcattaaccgggac atcctc-3'. For PPase (At1g13320): PPase-L, 5' -taacgtggcc aaaatgatgc-3', and PPase-R, 5' -gttctccacaaccgcttggt-3'. PCR products were visualized on an agarose gel stained with ethidium bromide. Fragment sizes for SGR1 (141 bp), SAG12 (93 bp), and PPase (61 bp) were all of the expected size.

\section{Sample preparation, probe labelling and protein analysis}

Probes were synthesised previously: DCG-04 [50], AMS101 [51] and MV151 [43]. Proteins were extracted from $100 \mathrm{mg}$ of homogenised frozen leaves in $0.5 \mathrm{ml}$ water for DCG-04 labeling or $100 \mu \mathrm{L}$ water for labeling with other probes. Debris was removed by centrifugation (2 min at $16000 \mathrm{~g}$ ). Labelling was conducted in $60 \mu \mathrm{l}$ of protein extract containing $70 \mathrm{mM}$ sodium acetate buffer (NaOAc) with probe-dependent $\mathrm{pH}, 1 \mathrm{mM}$ DTT and 0.2 or $2 \mu \mathrm{M}$ DCG-04, $2 \mu \mathrm{M}$ AMS101 and $2 \mu \mathrm{M}$ MV151. Extracts labelled with DCG-04 were incubated for 5 hours at room temperature $\left(22-25{ }^{\circ} \mathrm{C}\right)$ with continuous mixing, while samples labelled with fluorescent probes AMS101 and MV151 were incubated for 2 hours at room temperature in the dark. Equal dilutions of DMSO were added to the no-probe controls. Preincubation with $1 \mathrm{mM}$ E-64 added as a control for samples incubated with DCG-04 and MV151 for detection of PLCPs. The labelling reaction was stopped by adding $4 \mathrm{x}$ SDS-PAGE loading-buffer containing $\beta$-mercaptoethanol and then proteins were separated by $12 \%$ SDS PAGE. Labelled proteins were visualized by in-gel fluorescence scanning using a Typhoon 9000 scanner (GE Healthcare Life Science, http://www.gelifesciences.com) with excitation and emission at 532 and $580 \mathrm{~nm}$ respectively, or transferred to a membrane and analysed using streptavidin-HRP. Anti-RD21 and anti-AALP antibodies were described previously $[25,52]$.
Affinity purification and identification of labelled proteins Selected leaves of 6-week old plants growing in 12/ 12 hour light conditions were covered with aluminium foil for 7 days to induce senescence. Proteins were then extracted from five leaves into $2-4 \mathrm{~mL}$ water with subsequent centrifugation for $5 \mathrm{~min}$ at $20000 \mathrm{~g}$. Supernatant was diluted with $1 \mathrm{M}$ labelling buffer (1 M NaOAc, $\mathrm{pH}$ 6) to the final concentration of $50 \mathrm{mM}$ and protein concentration of $5 \mathrm{mg} / \mathrm{mL}$. The protein extract was labelled with $1 \mu \mathrm{M}$ DCG-04 in the presence of $1 \mathrm{mM}$ DTT for 2 hours at room temperature with gentle agitation. The labelled protein extract was applied to a PD10 column that had been equilibrated with $50 \mathrm{mM}$ Tris- $\mathrm{HCl}, \mathrm{pH}$ 8. Desalted samples were incubated with $100 \mu \mathrm{l}$ avidin beads for 1 hour under gentle agitation. Avidin beads were collected by centrifugation for $5 \mathrm{~min}$ at $2000 \mathrm{rpm}$. Beads were washed twice with 1\% SDS and twice with $6 \mathrm{M}$ Urea, once with $50 \mathrm{mM}$ Tris pH 8, once with $0.1 \%(\mathrm{w} / \mathrm{v})$ Tween 20 and once with water. Beads were then incubated in $100 \mathrm{mM}$ DTT for 20 min under gentle agitation, followed by incubation in $100 \mathrm{mM}$ iodoacetamide under gentle agitation in the dark for $20 \mathrm{~min}$. After washing three times in water, loading buffer was added to the beads and proteins separated by $12 \%$ SDS-PAGE. Labelled proteins in gels were visualized by Sypro Ruby staining. The visualized protein bands were excised and placed into $1.5 \mathrm{ml}$ Eppendorf tubes. The slices were washed with $500 \mathrm{ml}$ of $100 \mathrm{mM}$ ammonium bicarbonate (Sigma) twice for $15 \mathrm{~min}$. Proteins were reduced with Tris(2-carboxyethyl)-phospine (Sigma) for $30 \mathrm{~min}$ at $62^{\circ} \mathrm{C}$ and alkylated with $55 \mathrm{mM}$ iodoacetamide for $30 \mathrm{~min}$ at room temperature. Gel fragments were washed three times for $15 \mathrm{~min}$ in 50:50 acetonitrile: $100 \mathrm{mM}$ ammonium bicarbonate and dehydrated with $50 \mu \mathrm{l}$ of $100 \%$ acetonitrile. Acetonitrile was removed and gel fragments were dried using an Eppendorf SpeedVac for 5 minutes. Gel slices were incubated in $25 \mathrm{mM}$ ammonium bicarbonate and $10 \mathrm{ng} \mu \mathrm{L}^{-1}$ trypsin overnight at $37{ }^{\circ} \mathrm{C}$. The supernatant was transferred to a new tube and gel slices were treated with 5\% formic acid for $15 \mathrm{~min}$ at room temperature to inactivate trypsin. Gel slices were washed three times with $100 \%$ acetonitrile for $5 \mathrm{~min}$. All supernatants were combined and concentrated in an Eppendorf SpeedVac to a final volume of approximately $10 \mu$ l. Tryptic peptides were analysed using a Thermo Scientific LTQ XL mass spectrometer according to [53].

\section{Results}

PLCPs and VPEs are amongst the major senescenceinduced genes in leaves

To select proteases implicated in leaf senescence, we compared the transcript levels for Arabidopsis proteaseencoding genes in green and senescent leaves from a 
recently published leaf development time course [54]. We binned these proteases into 41 protease families according to the MEROPS peptidase database [7]. On average, the highest transcript levels in senescent leaves (>1000 fragments per kilobase per million, FPKM) were observed for Aspartic proteases (clan AA, family A1), PLCPs (clan CA, family C1A), VPEs (clan CD, family C13), and Clp endopeptidases (clan SK, family S14) (Additional file 2: Figure S1A). We focused our attention to VPEs and PLCPs because those families contained the most senescence-induced protease genes, and because their average expression change was higher than 2 fold, and we have tools to monitor their activity.

The largest increase in transcript level in the PLCP C1A group was for SAG12, which showed a 1934-fold induction, dominating the PLCP transcript levels at $2145 \pm 690$ FPKM (Additional file 2: Figure S1B and Additional file 3: Table S1), consistent with SAG12 being a major senescence-specific marker gene [18-20]. Besides $S A G 12$, transcript levels in senescent leaves were also high and induced more than two-fold for $R D 21 A$ (3.7-fold, $945 \pm$ FPKM), CTB3 (9.5-fold, $427 \pm 108$ FPKM), RD19A (2.1-fold, $814 \pm 73$ FPKM), RD19C (2.6fold, $1038 \pm 42$ FPKM) and AALP (3.0-fold, $721 \pm 96$ FPKM) (Additional file 2: Figure S1B and Additional file
3: Table S1). However, at the overall mRNA level, transcripts of RD21A, SAG12, RD19A, RD19C, AALP and CTB3 dominated the PLCP transcriptome of senescent leaves. Transcript levels of all $V P E$ genes are upregulated during senescence but only levels of $\gamma V P E$ transcripts were relatively high $(963 \pm 100$ FPKM) in the transcriptome of senescing leaves (Additional file 2: Figure S1B and Additional file 3: Table S1).

\section{Senescing leaves have increased PLCP and decreased VPE activities}

To induce senescence in Arabidopsis, we individually darkened leaves of approximately 8-week-old Arabidopsis plants (grown in 12/12 and 8/16 hours day/night light cycles, respectively) by covering leaves with aluminium foil for up to seven days [55] (Fig. 1a). Aluminium foil was lined inside with dark plastic and the leaves remained attached to the plant. The five to six largest leaves with approximately the same size and age were chosen for covering on each plant. This system resembles shading in nature and induces natural degradation of chlorophyll and the large subunit of Rubisco (Fig. 1b and c). Under these conditions, the expression of senescence marker gene SAG12 and SGR1 (Stay Green Gene 1, [56]) were induced (Fig. 1d), demonstrating that this
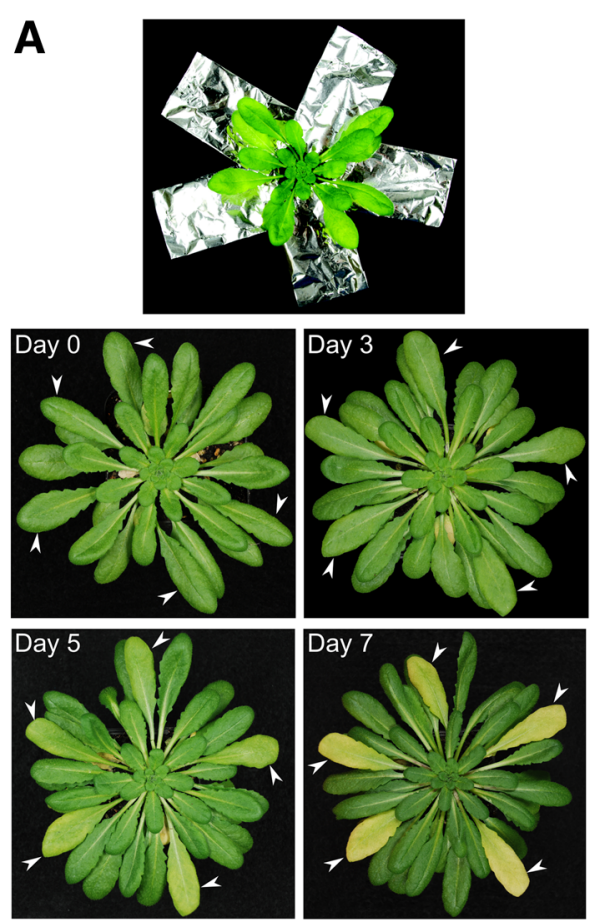

B

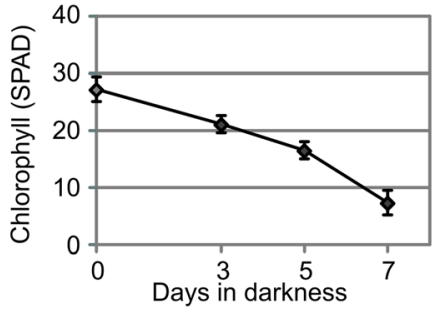

C

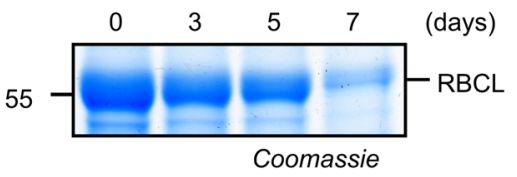

D

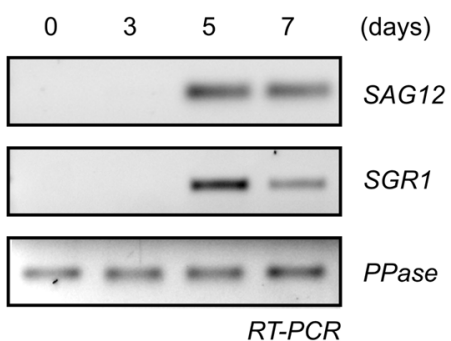

Fig. 1 Individually darkened leaves on intact plants. a Five individually darkened leaves covered with aluminium foil. Arabidopsis plants (Col-0) were grown under short day conditions (8/16 hours day/night cycles). b Changes in chlorophyll ratio in individually darkened leaves. Each point represents the mean of means of 5 leaves from 4 individual plants with standard deviation (c) Changes in abundance of the large subunit of Rubisco (RBCL) in individually darkened leaves. d Expression of SAGs markers in individually darkened leaves: SAG12, SGR1 (Stay Green Gene 1), and PPase (Protein Phosphatase 2A Subunit A3, control) 
treatment induces the classical senescence program. We used these individually darkened leaves for subsequent experiments.

To study the activity of PLCPs during leaf senescence by $A B P P$, we labeled leaf extracts of individually darkened leaves with DCG-04, a biotinylated chemical probe based on PLCP inhibitor E-64 [50, 57]. Detection of biotinylated proteins revealed increased intensities of signals migrating at 25,30 and $40 \mathrm{kDa}$, which represent AALP, mature $(\mathrm{m})$ and intermediate (i) RD21A, respectively (Fig. 2a, [43, 57]). Preincubation with an excess of E-64 prevented labeling of these proteins, suggesting that the signals represent PLCPs (Additional file 2: Figure S2). To confirm the increased PLCP labeling, we labeled the same proteomes with MV151, a fluorescent probe that can label a subset of the PLCPs, including RD21A [43]. MV151 labeling displayed increased intensities of 30 and $40 \mathrm{kDa}$ signals, which are likely to represent mRD21A and iRD21A, respectively (Fig. 2b, [43]). To study accumulation of RD21A and AALP proteins, we performed western analysis using RD21A and AALP antibodies [25, 52]. Consistent with the increased labeling, we found that RD21A and AALP proteins also accumulate in individually darkened leaves, concomitantly with decreasing amounts of the large subunit of Rubisco (Fig. 2c and d).

To monitor the activity of VPEs in individually darkened leaves we labeled leaf extracts with AMS101, a fluorescent activity-based probe for VPEs [51]. AMS101 detected signals at 40 and $43 \mathrm{kDa}$, which likely represent immature and mature isoforms of $\gamma \mathrm{VPE}$ because this causes the major VPE activity in green leaves (Fig. 2e, [51]), and $\gamma V P E$ transcript level dramatically increases in senescent leaves (Additional file 2: Figure S1B). However, the intensity of this signal decreased during senescence (Fig. 2e), despite upregulated VPE transcript levels.

\section{Many PLCPs have increased activity in senescing leaves}

To identify the proteins labelled with DCG-04 extracted from individually darkened senescent leaves, leaf extracts generated at days 0 and 7 were labeled with and without DCG-04 and biotinylated proteins were purified and separated on protein gels. Biotinylated proteins increased dramatically in abundance at day 7 when compared to day- 0 control leaves, and most signals were absent in the no-probe-controls (Fig. 3a). Eight protein band regions were excised, treated with trypsin, and analysed by mass spectrometry. Peptides from eleven PLCPs were
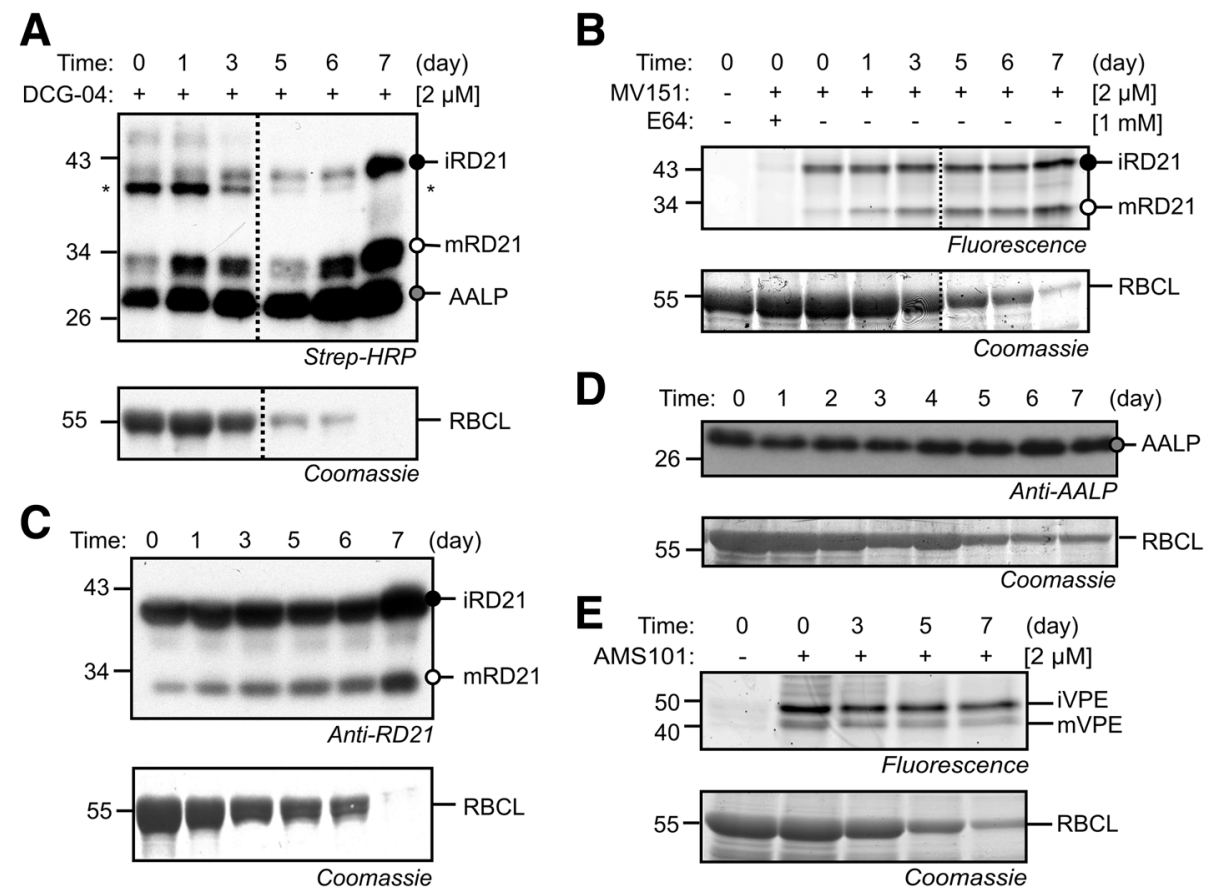

Fig. 2 Induced PLCP and reduced VPE activities individually darkened leaves. Increased DCG-04 (a) and MV151 (b) labeling of PLCPs during senescence. Leaf extracts of equal fresh weights of individually darkened leaves were labeled for 5 hours with $2 \mu \mathrm{M}$ DCG-04 at pH 6.5 or $2 \mu \mathrm{M}$ MV151 at pH 4.5 and biotinylated proteins were detected using streptavidin-HRP (a) or fluorescent proteins were detected by scanning (b), respectively. * , endogenously biotinylated protein. $\mathbf{c}$, d Accumulation of RD21A (c) and AALP (d) proteins in individually darkened leaves. Protein extracts of equal fresh weights of individually darkened leaves were separated and detected from protein blots using RD21A and AALP -specific antibodies, respectively. e Reduced AMS101 labeling of VPEs in individually darkened leaves. Leaf extracts of equal fresh weights of individually darkened leaves were labeled for 2 hours with 2 MM AMS101 at pH5.5 and analysed by fluorescence scanning. Coomassie stains of the membranes (a-d) or protein gel (e) is used as a control to show degradation of the large subunit of Rubisco (RBCL). The dotted line $(\mathbf{a}, \mathbf{b})$ indicates a removed lane from a western blot 


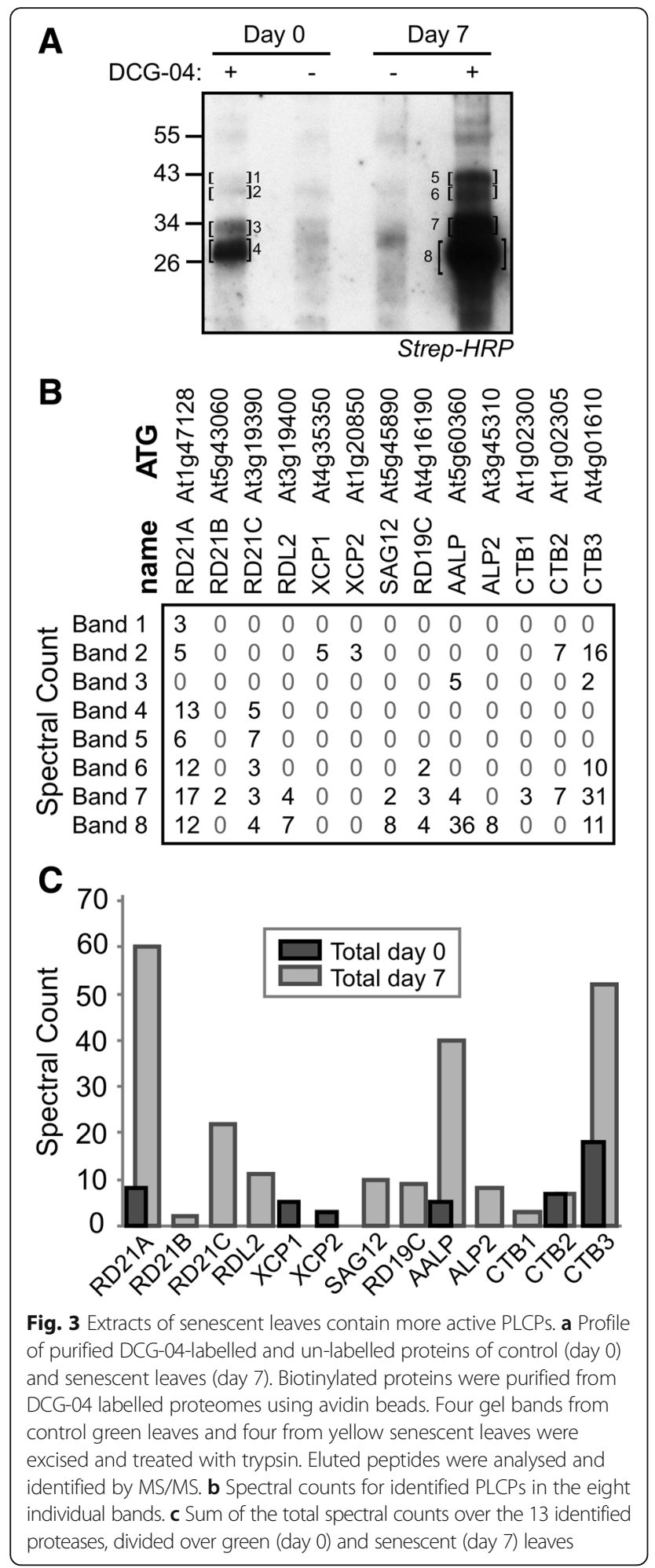

detected, including SAG12 (Fig. 3b). The highest number of spectral counts in senescent leaves were from RD21A, followed by CTB3, AALP, RD21C, RDL2, SAG12, RD19C, ALP2, CTB2, CTB1 and RD21B (Fig. 3c). Notably, peptides from XCP1 and XCP2 were detected only in green leaves and were not identified in senescent leaves (Fig. 3c), consistent with reduced transcript levels (Additional file 2: Figure S1B). All other detected proteases seem to have higher activity levels in senescing leaves (Fig. 3c).

\section{RD21A and AALP are the dominant active PLCPs in senescing leaves}

To confirm the identity of the proteases causing the major signals in the DCG-04 activity profile of senescent leaves, we labeled leaf extracts of green and senescent leaves with DCG-04 of the $\operatorname{sag} 12-1, r d 21 A-1, c t b 3-1$ and aalp-1 null mutants [42]. We only detected an altered protease activity profile for $r d 21 A-1$ and aalp-1 mutants (Fig. 4a). The 40 signal was absent and the $30 \mathrm{kDa}$ signal strongly reduced in the $r d 21 A-1$ mutant and the $25 \mathrm{kDa}$ signal was missing in the aalp-1 mutant, indicating that these signals are caused by RD21A and AALP, respectively. Consistently, the $r d 21 \mathrm{~A}-1 /$ aalp-1 double mutant lacks all three major signals (Fig. 4b), indicating that RD21A and AALP are the major PLCP activities in

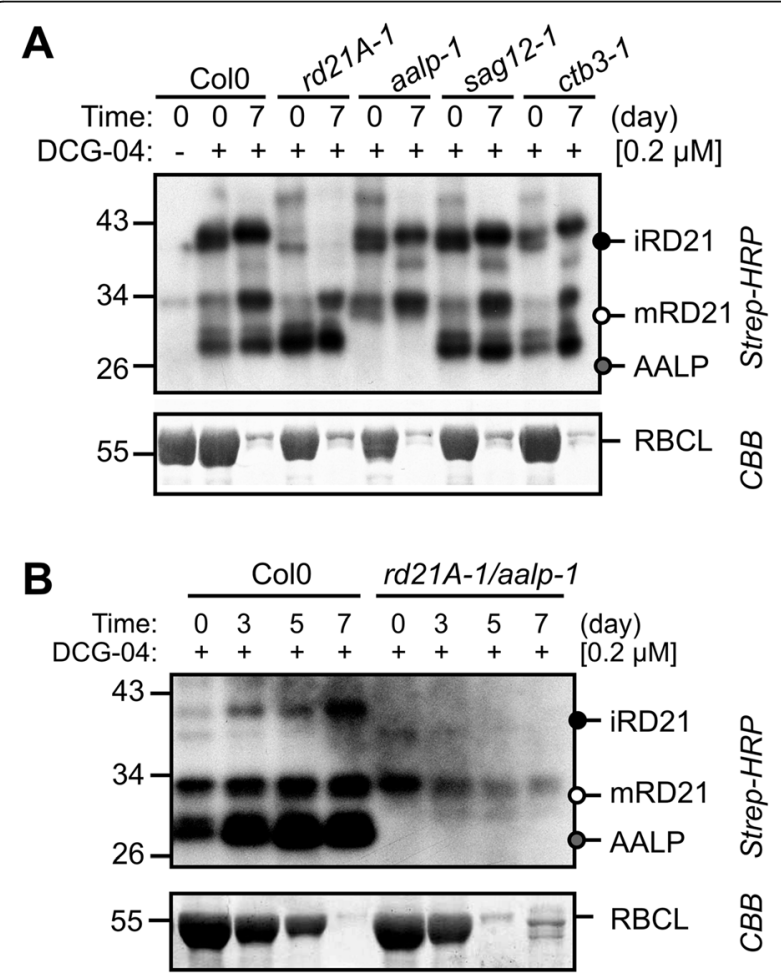

Fig. 4 Major PLCP activities are depleted in senescent leaves of rd21A-1/aalp-1 double mutant plants without affecting Rubisco levels. a PLCP activity profiles of control (day 0 ) and senescent leaf (day 7) of rd21A-1, aalp-1, sag12-1 and ctb3-1 mutants and wild-type plants. b PLCP activity profiles of individually darkened leaves at $0,3,5$ and 7 days of the $r d 21 \mathrm{~A}-1 /$ aalp-1 double mutant in comparison to wild-type (Col0) plants. Leaf extracts of equal fresh weights of individually darkened leaves were labeled for 5 hours with $0.2 \mu \mathrm{M} \mathrm{DCG-04}$ at pH 6.5 and biotinylated proteins were detected from protein blots using streptavidin-HRP 
senescing leaves, and that no other PLCP compensates for the reduced PLCP activity in this double mutant. Importantly, despite the absence of major PLCP activities in these plants, the decline of rubisco levels is not reduced in the $r d 21 A-1 /$ aalp-1 mutant plants (Fig. 4b, bottom).

\section{PLCP and VPE protease mutants do not have a strong senescence phenotype}

To study the contribution of PLCPs and VPEs to senescence in individually darkened leaves further, we subjected the following PLCP mutant lines to senescence assays: $r d 21 A-1$, aalp-1, sag12-1, ctb3-1, rd21A-1/aalp-1 double mutant, and $c t b 1 / c t b 2 / c t b 3$ triple mutant [42, 46]. We also included the RD21A overexpressor under the control of the $35 \mathrm{~S}$ promoter (35S::RD21A, Additional file 2: Figure S3). In addition, we included the quadruple VPE null mutant (qvpe) which lacks all four VPEs [45] and an overexpressor of $\gamma \mathrm{VPE}$ (35S:: $\gamma \mathrm{VPE}$, [44]) which might prevent the $\gamma \mathrm{VPE}$ decline during senescence.

We measured the chlorophyll ratio in leaves individually darkened for seven days after the plants were grown under short day conditions for eight weeks. The data did not show any significant difference between tested lines and their wild-type control (Fig. 5). However, this senescence assay displays senescence of individual leaves but not the whole plant. We therefore expanded our senescence assays to plants grown under long day conditions (16/8 hours day/night cycles) to study natural senescence of rosette leaves induced upon flowering. We monitored the number of green and senescent leaves at different time points during development under long day conditions. Interestingly, the aalp-1 and $r d 21 A-1 /$ aalp-1 mutants showed significantly more green leaves and less senescent leaves at early stages of developmental senescence than wild-type plants (Fig. 6a and b). By

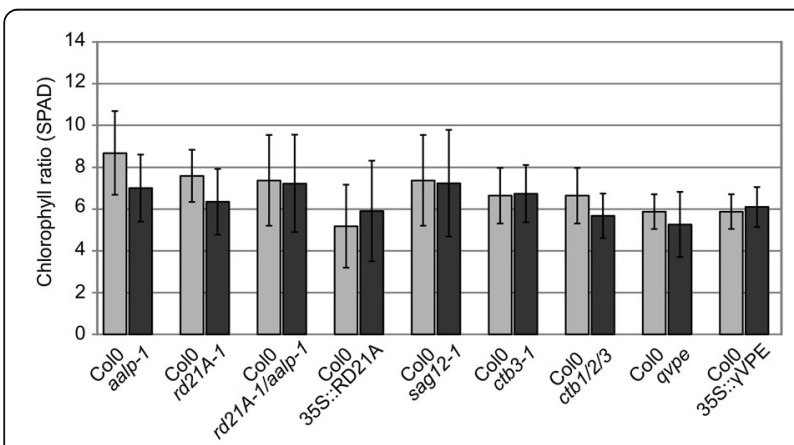

Fig. 5 Chlorophyll ratio is unaltered in individually darkened leaves for Cys protease mutant- and overexpressor lines. Chlorophyll ratio was measured with a SPAD meter on at least six leaves covered with aluminium foil for seven days from a total of eight plants. Error bars represent standard deviation of $n=8$ biological replicates contrast, other mutants ( $r d 21 A-1$, sag12-1, ctb3-1, ctb1/ $2 / 3$ and qvpe mutants) and lines overexpressing RD21A or $\gamma \mathrm{VPE}$ (35S::RD21A and 35S:: $\gamma \mathrm{VPE}$ ) did not show significant differences in the number of green and senescent leaves (Additional file 2: Figure S4). The fact that both aalp-1 and $r d 21 A-1 /$ aalp-1 mutants, but not the rd21A-1 mutant, showed this whole plant senescence phenotype indicates that the aalp-1 mutation correlates with this delayed progression of the senescence phenotype. We were unable to identify independent aalp-1 null mutant alleles for verification of this phenotype. Thus at this stage we cannot exclude that the senescence phenotype is caused by the absence of AALP or originates from a secondary, unidentified mutation that co-segregated into the $r d 21 A-1 /$ aalp-1 double mutant.

\section{Discussion}

In this study we showed that, while PLCP and $\gamma V P E$-encoding genes are induced transcriptionally during senescence, ABPP probes showed that only PLCPs had increased activity in individually darkened leaves of Arabidopsis. Yamada et al. [25] previously showed that RD21A protein levels increase during developmental senescence of Arabidopsis. Increasing activities of PLCPs using the DCG-04 probe have also previously been observed during developmental leaf senescence in Arabidopsis and in wheat leaf-segments incubated in the dark $[57,58]$. However the identity of these active proteases in senescent Arabidopsis leaves was not previously known. In this work, 11 active proteases were purified and identified from senescent leaves: RD21A, CTB3, AALP, RD21C, RDL2, SAG12, RD19C, ALP2, CTB2, CTB1 and RD21B. Four detected PLCPs (SAG12, RD19C, CTB2 and CTB1) have not previously been detected by DCG-04 labelling in green leaves or other Arabidopsis organs [57, 59]. Our data demonstrate that these proteases are active in extracts of senescent leaves.

We have analysed mutants for senescence-associated PLCPs and $\gamma \mathrm{VPE}$ proteases for senescence phenotypes, as chlorophyll content in individually darkened leaves and the number of green and senescent leaves in naturally senescing plants. Surprisingly, none of the mutant lines showed any phenotype in individually darkened leaves, despite the evident lack of major protease activities displayed by DCG-04 labeling in these lines. The following lines did also not show any obvious phenotype during developmental senescence: $r d 21 A-1$, sag12-1, ctb3-1, ctb1/2/3, qvpe, 35S::RD21A and 35S:: $\gamma V P E$, consistent with previously published results for sag12 and $c t b 3$ mutants [2, 45].

The fact that single PLCP and VPE mutants showed no senescence phenotype may be due to redundancy between proteases. For instance, Arabidopsis CTB genes were reported to act redundantly in leaf senescence 

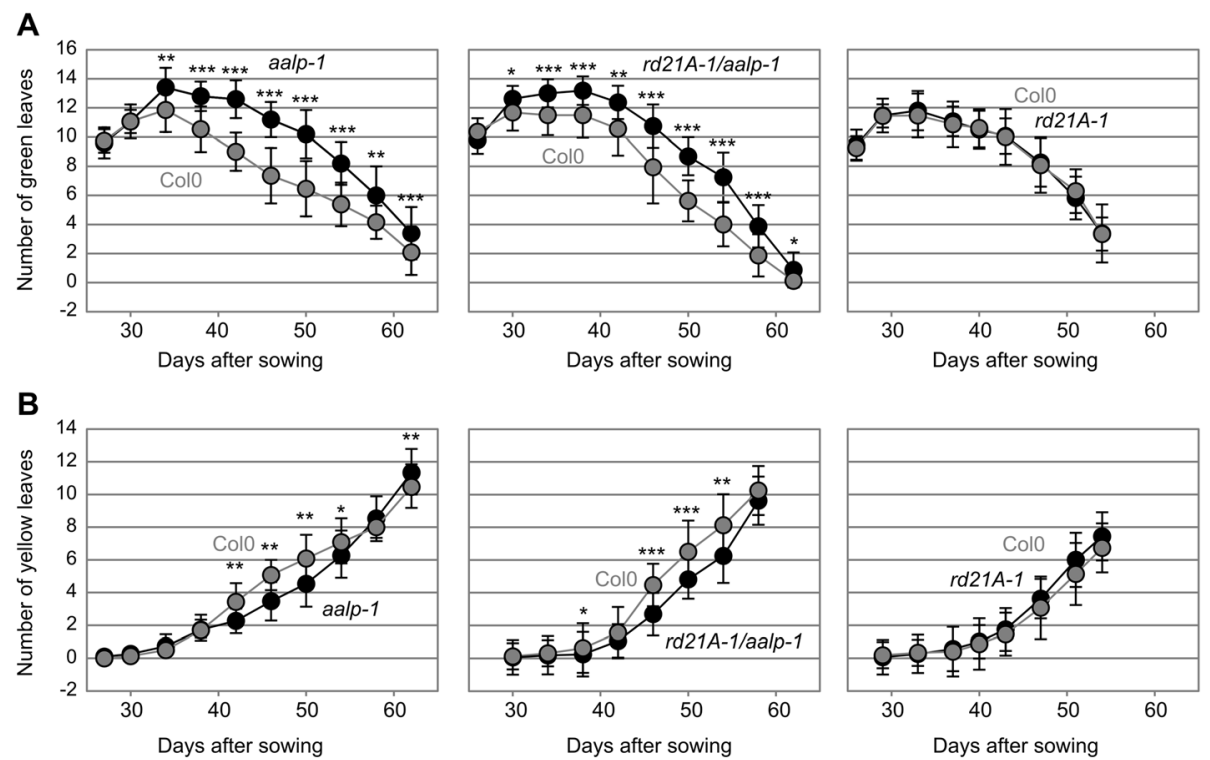

Fig. 6 Delayed onset of whole plant senescence in aalp-1 and rd21A-1/aalp-1 mutants. Plants were grown under long day conditions (16/8 day/ night). Number of green (a) and yellow (/senescent) (b) leaves at different time points after the sowing of wild-type plants and aalp-1, rd21A-1/ aalp-1 and rd21A-1 mutants. Each data point represents the mean of 16 plants with standard deviation from one representative experiment. The experiments were repeated twice with similar results

because only the triple $c t b 1 / 2 / 3$ mutant showed delayed senescence in detached leaves incubated in the dark [45]. In our senescence assay, however, the $c t b 1 / 2 / 3$ mutant has no senescence phenotype, possibly because different senescence assays induce different regulatory pathways [60]. For instance the $50 \mathrm{kDa}$ Rubisco cleavage fragment is present only in detached leaves and under low light but not in leaf segments exposed to high light and in intact plants induced to senesce by $\mathrm{N}$-deprivation [16]. Leaf senescence is also associated with the loss of water, so it is possible that the drought-responsive $R D 19 A$ and $R D 21 A$ genes [61] are upregulated at the late stages of dark-induced senescence due to the loss of the water, not because they are involved in the senescence process itself.

The delayed progression of senescence in aalp-1 and rd21A-1/aalp-1 mutants suggests that AALP contributes to the senescence process. Our observation is consistent with the report that suppression of the AALP orthologue in Broccoli delays senescence in florets [62]. AALP is a predicted aminopeptidase similar to mammalian cathepsin- $\mathrm{H}$. This protease cannot act as an endopeptidase because one side of the substrate binding groove is blocked by a minipeptide that originates from the prodomain and remains covalently bound through a disulphide bridge [63]. AALP shares these features and therefore probably acts on (neo) N-termini during the bulk protein degradation process in the vacuole. The delay in senescence in aalp-1 null mutants may be the result of an imbalance in amino acid availability.

\section{Conclusions}

Senescing Arabidopsis leaves show a massive transcriptional activation encoding Cys proteases, especially vacuolar processing enzymes (VPEs) and papain-like Cys proteases (PLCPs). Protease activity profiling demonstrates that in contrast to increased VPE transcript levels, VPE activity is not induced. By contrast, senescing leaves have an increased activity of PLCPs, and MS and mutant analysis show that this increased PLCP activity is dominated by RD21 and AALP. VPE and PLCP mutant and overexpressor lines do not show an altered rubisco degradation or chlorophyll ratio phenotypes. In whole plant senescence assays, however, aalp-1 and aalp-1/rd21-1 mutants show a delayed senescence, suggesting a role for AALP in developmental senescence. Taken together, these data indicate that Cys proteases play redundant roles in leaf senescence.

\section{Additional files}

Additional file 1: Table S2. Arabidopsis knock-out and over-expressor lines used in this study. (DOC $32 \mathrm{~kb}$ )

Additional file 2: Figure S1. Transcript levels of protease-encoding genes in mature and senescing leaves of Arabidopsis. (A) Transcript levels in FPKM of genes grouped per protease family. Transcript levels in FPKM were extracted from GSE43616 (Woo et al., 2016) and were summed up for each protease according to the protease families of the MEROPs database. (B) Transcript levels of genes encoding PLCPS (top) and VPEs (bottom). Shown are the transcript levels in mature green leafs (left, 16D $+18 D$ ), senescent leaves (middle, 28D+30D) and the ratio between green and senescence leaves (right). Error bars represent standard deviation (STDEV) of $(n=4)$ samples. Figure S2. E-64 suppresses DCG-04 labeling mature and senescent leaves. Leaf extracts of equal fresh weights of 
individually darkened leaves were preincubated with $2 \mu \mathrm{M}$ E-64 for 30 minutes and then labeled for 5 hours with $2 \mu \mathrm{M}$ DCG-04 at pH 6.5 and biotinylated proteins were detected using streptavidin-HRP or fluorescence scanning, respectively. ${ }^{*}$, endogenously biotinylated proteins. Figure S3. Characterization of the transgenic 35S::RD21 Arabidopsis line. The homozygous progeny of a Col-0 plant transformed with pRH628 carrying $355:: R D 21$ is compared to the wild-type (Col-0) and to two RD21 knock-out mutants: rd21-1 and rd21-2. Leaf extracts were labeled with DCG-04 and biotinylated proteins were detected on protein blots using streptavidin-HRP. Figure S4. No altered natural senescence in other PLCPNPE mutants and overexpressor lines. Number of green leaves at different time points of wild-type and mutant plants grown at long days. Error bars represent STDEV of $n=16$ biological replicates. (PDF $469 \mathrm{~kb}$ )

Additional file 3: Table S1. Transcript levels of proteases in non-senescent and senescent leaves. (XLSX $24 \mathrm{~kb}$ )

\section{Abbreviations}

AALP: Arabidopsis aleurain-like protease, ALP, aleurain-like protease; ABPP: Activity-based protein profiling; CTB: Cathepsin B; PAO:

Pheophorbide-a-oxygenase; PLCP: Papain-like Cys protease; RD19: Responsiveto-desiccation 19; RD21: Responsive-to-desiccation 21; RDL: RD21-like protease; SAG12: Senescence-associated gene 12; SGR1: Stay Green Gene 1; VPE: Vacuolar processing enzyme; XCP: Xylem-specific protease

\section{Acknowledgements}

We would like to thank Dr. Ikuko Hara-Nishimura for providing the RD21A antibody; Dr. Natasha Raikhel for the AALP antibody and the 35S:::VVPE line; Dr. Darren Gruis for the qvpe quadrupole mutant; Dr. Garry Loake for providing ctb1/2/3 triple mutant; Dr. Hermen Overkleeft for providing DCG-04 and MV151; and Dr. Matthew Bogyo for providing AMS101.

\section{Funding}

This research was financially supported by the Humboldt Foundation, Marie Curie Postdoctoral Fellowship, the Max Planck Society and the University of Oxford. RvdH is supported by ERC Consolidator grant 616449 'GreenProteases', and AP and AHM were supported by the Australian Research Council (DE120102913; CE140100008; FT110100242). The funding bodies had no role in the design of the study and the collection, analysis and interpretation of data and in writing the manuscript.

\section{Availability of data and materials}

Seeds and activity-based probes will be provided upon request.

\section{Authors' contributions}

AP and RvdH designed the experiments; AP performed the experiments; TS selected T-DNA knock-out lines; RT performed growth assays; SN and FK performed MS analysis; AP, HM and RvdH wrote the manuscript with input of the other authors. All authors read and approved the final manuscript.

\section{Competing interests}

The authors declare that they have no competing interests.

\section{Consent for publication}

Not applicable.

\section{Ethics approval and consent to participate}

Not applicable.

\section{Author details}

${ }^{1}$ The Plant Chemetics laboratory, Max Planck Institute for Plant Breeding Research, 50829 Cologne, Germany. ${ }^{2}$ The Skaggs Institute for Chemical Biology and Department of Chemical Physiology, The Center for Physiological Proteomics, The Scripps Research Institute, La Jolla 92037, California, USA. ${ }^{3}$ The Australian Research Council Centre of Excellence in Plant Energy Biology, The University of Western Australia, Perth, WA, Australia. ${ }^{4}$ The Plant Chemetics Laboratory, Department of Plant Sciences, University of Oxford, OX1 3RB Oxford, UK. ${ }^{5}$ Department of Plant Developmental Biology, Max Planck Institute for Plant Breeding Research, 50829 Cologne, Germany.
Received: 8 July 2016 Accepted: 19 December 2016

Published online: 06 January 2017

\section{References}

1. Xie Q, Michaeli S, Peled-Zehavi H, Galili G. Chloroplast degradation: one organelle, multiple degradation pathways. Trends Plant Sci. 2015;20:264-5.

2. Otegui MS, Noh YS, Martínez DE, Vila Petroff MG, Staehelin LA, Amasino RM Guiamet JJ. Senescence-associated vacuoles with intense proteolytic activity develop in leaves of Arabidopsis and soybean. Plant J. 2005;41:831-44.

3. Wada S, Ishida H, Izumi M, Yoshimoto K, Ohsumi Y, Mae T, Makino A. Autophagy plays a role in chloroplast degradation during senescence in individually darkened leaves. Plant Physiol. 2009;149:885-93.

4. Wang S, Blumwald E. Stress-induced chloroplast degradation in Arabidopsis is regulated via a process independent of autophagy and senescenceassociated vacuoles. Plant Cell. 2014;26:4875-88.

5. Springer A, Kang C, Rustgi S, von Wettstein D, Reinbothe C, Pollmann S, Reinbothe S. Programmed chloroplast destruction during leaf senescence involves 13-lipoxygenase (13-LOX). Proc Natl Acad Sci U S A. 2016;113:3383-8.

6. Bhalerao R, Keskitalo J, Sterky F, Erlandsson R, Björkbacka H, Birve SJ, Karlsson J, Gardeström P, Gustafsson P, Lundeberg J, Jansson S. Gene expression in autumn leaves. Plant Biol. 2003;131:430-42.

7. Rawlings ND, Barrett AJ, Finn R. Twenty years of the MEROPS database of proteolytic enzymes, their substrates and inhibitors. Nucl Acid Res. 2016:44:D343-50.

8. Diaz I, Martinez M. Plant C1A cysteine peptidases in germination and senescence. In: Rawlings ND, Salvesen G, eds, Handbook of proteolytic enzymes. Amsterdam: Academic Press, Elsevier. 2013. pp 1853-1858

9. Roberts IN, Caputo C, Criado MV, Funk C. Senescence-associated proteases in plants. Physiol Plant. 2012;145:130-9.

10. Gepstein S, Sabehi G, Carp MJ, Hajouj T, Nesher MF, Yariv I, Dor C, Bassani M. Large-scale identification of leaf senescence-associated genes. Plant J. 2003;36:629-42.

11. Guo Y, Cai Z, Gan S. Transcriptome of Arabidopsis leaf senescence. Plant Cell and Environment. 2004;27:521-49.

12. Fischer AM. The complex regulation of senescence. Crit Rev Plant Sci. 2012;31:124-47.

13. Gregersen PL, Holm PB. Transcriptome analysis of senescence in the flag leaf of wheat (Triticum aestivum L.). Plant Biotechn J. 2006;5:192-206.

14. Parrott DL, Martin JM, Fischer AM. Analysis of barley (Hordeum vulgare) leaf senescence and protease gene expression: a family of C1A cysteine proteases is specifically induced under conditions characterized by high carbohydrate but low moderate nitrogen levels. New Phytol. 2010;187:313-31.

15. Hollmann J, Gregersen PL, Krupinska K. Identification of predominant genes involved in regulation and execution of senescence-associated nitrogen remobilization in flag leaves of field grown barley. J Exp Bot. 2014;65:3963-73.

16. Thoenen M, Herrmann B, Feller U. Senescence in wheat leaves: is a cysteine endopeptidase involved in the degradation of the large subunit of Rubisco? Acta Physiol Plantarum. 2007;29:339-50.

17. Van der Hoorn RAL. Plant proteases: from phenotypes to molecular mechanisms. Annu Rev Plant Biol. 2008;59:191-223.

18. Hensel LL, Grbic V, Baumgarten DA, Bleecker AB. Developmental and agerelated processes that influence the longevity and senescence of photosynthetic tissues in Arabidopsis. Plant Cell. 1993;5:553-64.

19. Lohman KN, Gan S, Amasino J, Amasino RM, Manorama C. Molecular analysis of natural leaf senescence in Arabidopsis thaliana. Physiol Plant. 1994;92:322-8.

20. Noh YS, Amasino RM. Regulation of developmental senescence is conserved between Arabidopsis and Brassica napus. Plant Mol Biol. 1999;41:195-206.

21. Chen HJ, Huang DJ, Hou WC, Liu JS, Lin YH. Molecular cloning and characterization of a granulin-containing cysteine protease SPCP3 from sweet potato (Ipomoea batatas) senescent leaves. J Plant Physiol. 2006;163:863-76.

22. Chen HJ, Su CT, Lin CH, Huang GJ, Lin YH. Expression of sweet potato cysteine protease SPCP2 altered developmental characteristics and stress responses in transgenic Arabidopsis plants. J Plant Physiol. 2010;167:838-47.

23. Esteban-García B, Garrido-Cárdenas JA, Alonso DL, García-Maroto F. A distinct subfamily of papain-like cysteine proteinases regulated by senescence and stresses in Glycine max. J Plant Physiol. 2010;167:1101-8.

24. Coupe SA, Sinclair BK, Watson LM, Heyes JA, Eason JR. Identification of dehydration-responsive cysteine proteases during post-harvest senescence of broccoli florets. J Exp Bot. 2003;54:1045-56. 
25. Yamada K, Mtsushima R, Nishimura M, Hara-Nishimura I. A slow maturation of cysteine protease with a granulin domain in the vacuoles of senescing Arabidopsis leaves. Plant Physiol. 2001;127:1626-34.

26. Tajima T, Yamaguchi A, Matsushima S, Satoh M, Hayasaka S, Yoshimatsu K, Shioi Y. Biochemical and molecular characterization of senescence-related cysteine protease-cystatin complex from spinach leaf. Physiol Plant. 2011; 141:97-116.

27. Rojo E, Martín R, Carter C, Zouhar J, Pan S, Plotnikova J, Jin H, Paneque M, Sánchez-Serrano JJ, Baker B, Ausubel FM, Raikhel NV. VPEY exhibits a caspase-like activity that contributes to defense against pathogens. Curr Biol. 2004;14:1897-906

28. Hara-Nishimura I, Hatsugai N, Nakaune S, Kuroyanagi M, Nishimura M. Vacuolar processing enzyme: an executor of plant cell death. Curr Opin Plant Biol. 2005;8:404-8.

29. Hatsugai N, Kuroyanagi M, Nishimura M, Hara-Nishimura I. A cellular suicide strategy of plants: vacuole-mediated cell death. Apoptosis. 2006;11:905-11.

30. Hara-Nishimura I, Hatsugai N. The role of vacuole in plant cell death. Cell Death Diff. 2011;18:1298-304

31. Kinoshita T, Yamada K, Hiraiwa N, Kondo M, Nishimura M, Hara-Nishimura I. Vacuolar processing enzyme is up-regulated in the lytic vacuoles of vegetative tissues during senescence and under various stressed conditions. Plant J. 1999;19:43-53.

32. Muller GL, Drincovich MF, Andreo CS, Lara MV. Role of photosynthesis and analysis of key enzymes involved in primary metabolism throughout the lifespan of the tobacco flower. J Exp Bot. 2010;61:3675-88.

33. Solomon M, Belenghi B, Delledonne M, Menachem E, Levine A. The involvement of cysteine proteases and protease inhibitor genes in the regulation of programmed cell death in plants. Plant Cell. 1999;11:431-43.

34. Hayashi Y, Yamada K, Shimada T, Matsushima R, Nishizawa N, Nishimura M, Hara-Nishimura I. A proteinase-storing body that prepares for cell death or stresses in the epidermal cells of Arabidopsis. Plant Cell Physiol. 2001:42:894-9.

35. Tajima T, Yamaguchi A, Matsushima S, Satoh M, Hayasaka S, Yoshimatsu K, Shioi Y. Biochemical and molecular characterization of senescence-related cysteine protease-cystatin complex from spinach leaf. Physiol Plant. 2011:141:97-116.

36. Diaz-Mendoza M, Arroyo-Velasco B, Gonzalez-Melendi P, Martinez M, Diaz I. C1A cysteine protease-cystatin interactions in leaf senescence. J Exp Bot. 2014;65:3825-33.

37. Prins A, van Heerden PDR, Olmos E, Kunert KJ, Foyer $\mathrm{CH}$. Cysteine proteinases regulate chloroplast protein content and composition in tobacco leaves: a model for dynamic interactions with ribulose-1,5bisphosphate carboxylase/oxygenase (Rubisco) vesicular bodies. J Exp Bot. 2008:59:1935-50.

38. Lampl N, Budai-Hadrian O, Davydov O, Joss TV, Harrop SJ, Curmi PM, Roberts TH, Fluhr R. Arabidopsis AtSerpin1: crystal structure and in vivo interaction with its target protease responsive to desiccation-21 (RD21). J Biol Chem. 2010;285:13550-60.

39. Cravatt BF, Wright AT, Kozarich JW. Activity-based protein profiling: from enzyme chemistry to proteomic chemistry. Annu Rev Biochem. 2008;77:383-414

40. Kolodziejek I, Van Der Hoorn RAL. Mining the active proteome in plant science and biotechnology. Curr Opin Biotechn. 2010;21:225-33.

41. Morimoto K, Van der Hoorn RAL. The increasing impact of activity-based protein profiling in plant science. Plant Cell Physiol. 2016;57:446-61.

42. Wang Z, Gu C, Colby T, Shindo T, Balamurugan R, Waldmann H, Kaiser M, van der Hoorn RAL. Beta-lactone probes identify a papain-like peptide ligase in Arabidopsis thaliana. Nat Chem Biol. 2008;4:557-63.

43. Gu C, Shabab M, Strasser R, Wolters PJ, Shindo T, Niemer M, Kaschani F, Mach L, van der Hoorn RAL. Post-translational regulation and trafficking of the granulin-containing protease RD21 of Arabidopsis thaliana. PLoS One. 2012;7, e32422.

44. Rojo E, Zouhar J, Carter C, Kovaleva V, Raikhel NV. A unique mechanism for protein processing and degradation in Arabidopsis thaliana. Proc Natl Acad Sci U S A. 2003;100:7389-94.

45. Gruis D, Schulze J, Jung R. Storage protein accumulation in the absence of the vacuolar processing enzyme family of cysteine proteases. Plant Cell. 2004;16:270-90.

46. McLellan H, Gilroy EM, Yun BW, Birch PR, Loake GJ. Functional redundancy in the Arabidopsis cathepsin B gene family contributes to basal defence, the hypersensitive response and senescence. New Phytolt. 2009;183:408-18.
47. Ling Q, Huang W, Jarvis P. Use of a SPAD-502 meter to measure leaf chlorophyll concentration in Arabidopsis thaliana. Photosynth Res. 2011;107:209-14.

48. Levey S, Wingler A. Natural variation in the regulation of leaf senescence and relation to other traits in Arabidopsis. Plant Cell Environ. 2015;28:223-31.

49. Balazadeh S, Parlitz S, Mueller-Roeber B, Meyer RC. Natural developmental variations in leaf and plant senescence in Arabidopsis thaliana. Plant Biology. 2008;10:136-47.

50. Greenbaum DC, Baruch A, Grainger M, Bozdech Z, Medzihradszky KF, Engel J, DeRisi J, Holder AA, Bogyo M. A role for the protease Falcipain 1 in host cell invasion by the human malaria parasite. Science. 2002;298:2002-6.

51. Misas-Villamil JC, Toenges G, Kolodziejek I, Sadaghiani AM, Kaschani F, Colby T, Bogyo M, van der Hoorn RAL. Activity profiling of vacuolar processing enzymes reveals a role for VPE during oomycete infection. Plant J. 2013;73:689-700.

52. Ahmed SU, Rojo E, Kovaleva V, Venkataraman S, Dombrowski JE, Matsuoka K, Raikhel NV. The plant vacuolar sorting receptor AtELP is involved in transport of $\mathrm{NH}(2)$-terminal propeptide-containing vacuolar proteins in Arabidopsis thaliana. J Cell Biol. 2000;149:1335-44.

53. Kaschani F, Gu C, Niessen S, Hoover H, Cravatt BF, Van der Hoorn RAL. Diversity of serine hydrolase activities of non-challenged and Botrytisinfected Arabidopsis thaliana. Mol Cell Proteomics. 2009;8:1082-93.

54. Woo HR, Koo HJ, Kim J, Jeong H, Yang IO, Lee IH, Jun JH, Choi SH, Park SJ, Kang B, Kim YW, Phee BK, Kim JH, Seo C, Park C, Kim SC, Park S, Lee B, Lee S, Hwang D, Nam HG, Lim PO. Programming of plant leaf senescence with temporal and inter-organellar coordination of transcriptome in Arabidopsis. Plant Physiol. 2016;171:452-67.

55. Weaver LM, Amasino RM. Senescence is induced in individually darkened Arabidopsis leaves, but inhibited in whole darkened plants. Plant Physiol. 2001;127:876-86.

56. Aubry S, Mani J, Hörtensteiner S. Stay-green protein, defective in Mendel's green cotyledon mutant, acts independent and upstream of pheophorbide a oxygenase in the chlorophyll catabolic pathway. Plant Mol Biol. 2008;67:243-56.

57. Van der Hoorn RAL, Leeuwenburgh MA, Bogyo M, Joosten MH, Peck SC. Activity profiling of papain-Like cysteine proteases in plants. Plant Physiol. 2004;135:1170-8.

58. Martínez DE, Bartoli CG, Grbic V, Guiamet JJ. Vacuolar cysteine proteases of wheat (Triticum aestivum L.) are common to leaf senescence induced by different factors. J Exp Bot. 2007;58:1099-107.

59. Richau KH, Kaschani F, Verdoes M, Pansuriya TC, Niessen S, Stüber K, Colby T, Overkleeft HS, Bogyo M, Van der Hoorn RAL. Subclassification and biochemical analysis of plant papain-like cysteine proteases displays subfamily-specific characteristics. Plant Physiol. 2012;158:1583-99.

60. Van der Graaff E, Schwacke R, Schneider A, Desimone M, Flugge UI, Kunze R. Transcription analysis of Arabidopsis membrane transporters and hormone pathways during developmental and induced leaf senescence. Plant Physiol. 2001;141:776-92.

61. Yamaguchi-Shinozaki K, Koizumi M, Urao S, Shinozaki K. Molecular cloning and characterization of 9 cDNAs for genes that are responsive to desiccation in Arabidopsis thaliana: sequence analysis of one cDNA clone that encodes a putative transmembrane channel protein. Plant Cell Physiol. 1993:33:217-24.

62. Eason JR, Ryan DJ, Watson LM, Hedderley D, Christey MC, Coupe SA. Suppression of the cysteine protease, aleurain, delays floret senescence in Brassica oleracea. Plant Mol Biol. 2005;57:645-57.

63. Vasiljeva $O$, Dolinar M, Turk V, Turk B. Recombinant human cathepsin H lacking the mini chain is an endopeptidase. Biochem. 2003:42:13522-8. 